\title{
高熵陶瓷固溶结构的透射电镜研究
}

\author{
郭晓杰 ${ }^{1,3}$, 鲍伟超 ${ }^{1}$, 刘吉轩 ${ }^{2}$, 王新刚 ${ }^{1}$, 张国军 ${ }^{2}$, 许钫钫 ${ }^{1}$
}

(1. 中国科学院 上海硅酸盐研究所, 高性能陶瓷和超微结构国家重点实验室, 上海 200050; 2. 东华大学, 功能材 料研究所, 纤维材料改性国家重点实验室, 上海 200051; 3. 中国科学院大学, 材料科学与光电子功能中心, 北京 100049)

摘 要: 高熵会带来热力学上的高熵效应、结构上的晶格畸变效应、动力学上的迟滞扩散效应以及性能上的“鸡尾酒” 效应，通过高熵设计来提高陶瓷材料的性能是目前研究的热点，而通过透射电镜揭示高熵结构及其与性能相关性 的研究还很缺乏。本研究以相应金属氧化物、碳化嗍和石墨为原材料, 在制备高熵嗍化物和高熵碳化物粉体的基础 上，利用放电等离子体烧结制备得到高熵(TiZrHfNbTa) $\mathrm{B}_{2}$ 和 $(\mathrm{TiZrHfNbTa}) \mathrm{C}$ 陶瓷。采用透射电子显微镜及其能谱分 析手段对两种高熵陶瓷进行了纳米尺度和原子尺度的结构表征，发现过渡金属元素固溶后保持了晶体结构的完整 性，五种元素分布均匀，但在原子尺度存在固溶元素的浓度振荡、原子离散和晶格应变。本工作获得的原子尺度的 固溶结构信息将有助于对高熵陶瓷构效关系的理解，并为高熵陶瓷的组分和结构设计提供实验依据。

关 键 词: 高熵陶瓷; 透射电镜; 纳米尺度结构; 原子尺度结构

中图分类号: O766 文献标识码: A

\section{Study on the Solid Solution Structures of High-entropy Ceramics by Transmission Electron Microscopy}

\author{
GUO Xiaojie $^{1,3}$, BAO Weichao ${ }^{1}$, LIU Jixuan ${ }^{2}$, WANG Xingang ${ }^{1}$, ZHANG Guojun ${ }^{2}$, XU Fangfang ${ }^{1}$
}

(1. State Key Laboratory of High Performance Ceramics and Superfine Microstructure, Shanghai Institute of Ceramics, Chinese Academy of Sciences, Shanghai 200050, China; 2. State Key Laboratory for Modification of Chemical Fibers and Polymer Materials, Institute of Functional Materials, Donghua University, Shanghai 201620, China; 3. Center of Materials Science and Optoelectronics Engineering, University of Chinese Academy of Sciences, Beijing 100049, China)

\begin{abstract}
High-entropy brings high-entropy effect on thermodynamics, lattice distortion effect on structure, diffusion retardation effect on dynamics and "cocktail" effect on properties in materials. It is a hotspot to improve the properties of ceramics by high-entropy design. However, it still lacks the study of high-entropy structures and their correlation to the properties through transmission electron microscopy (TEM). In this study, high-entropy borides and carbides powders were fabricated by using metal oxides, boron carbide and graphite as raw materials. The high-entropy (TiZrHfNbTa)B $\mathrm{B}_{2}$ and (TiZrHfNbTa)C ceramics were then synthesized by spark plasma sintering
\end{abstract}

收稿日期: 2020-11-16; 收到修改稿日期：2020-12-28; 网络出版日期：2021-01-07

基金项目: 国家自然科学基金(52032001，51532009); 上海市青年科技英才扬帆计划(20YF1455500); 上海市科学技术委员 会科学基金会(18ZR1401400); 上海市无机材料测试与表征平台(19DZ2290700)

National Natural Science Foundation of China (52032001, 51532009); Shanghai Sailing Program (20YF1455500); Science and Technology Commission of Shanghai Municipality (18ZR1401400); Shanghai Technical Platform for Testing and Characterization on Inorganic Materials (19DZ2290700)

作者简介: 郭晓杰(1991-), 女, 博士研究生. E-mail: guoxiaojie20@mails.ucas.ac.cn GUO Xiaojie(1991-), female, PhD candidate. E-mail: guoxiaojie20@mails.ucas.ac.cn

通信作者: 鲍伟超, 助理研究员. E-mail: baoweichao@mail.sic.ac.cn; 张国军, 研究员. E-mail: gjzhang@dhu.edu.cn BAO Weichao, assistant professor. E-mail: baoweichao@mail.sic.ac.cn; ZHANG Guojun, professor. E-mail: gjzhang@dhu.edu.cn 
of the as-fabricated powders. Transmission electron microscope and energy dispersive spectrometry were used to characterize the structure of the two high-entropy ceramics at the nano-scale and atomic-scale. The integrity of crystal structure maintained after solid solution of five transition metal elements which were found to uniformly distribute in the ceramics. However, at atomic scales, concentration oscillations of solid solution elements, atomic dispersion and lattice strain were observed. The solid solution structures at atomic scales as-obtained in this work can help to understand the structure-property relationship of high-entropy ceramics and provide experimental basis for the composition and structure design of high-entropy ceramics.

Key words: high-entropy ceramics; transmission electron microscopy (TEM); structure in nano-scale; structure in atomic-scale

高熵概念来源于高熵合金, 它以多元组分固溶 来增加构型熵从而获得固溶体相结构上的稳定。高 熵会带来热力学上的高熵效应、结构上的晶格畸变 效应、动力学上的迟滞扩散效应以及性能上的“鸡尾 酒” 效应 ${ }^{[1]}$, 将高熵概念引入到陶瓷材料的研究已受

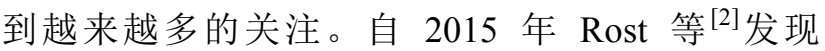
$(\mathrm{MgNiCoCuZn}) \mathrm{O}$ 高熵氧化物陶瓷中熵能驱动多相 和单相之间的可逆变化之后, 高熵氧化物 ${ }^{[3-7]}$ 、高 熵硼化物 ${ }^{[8-11]}$ 、碳化物 ${ }^{[2-15]}$ 、硅化物 ${ }^{[16-18]}$ 、MAX 相 ${ }^{[19-20]}$ 、阴离子高熵陶瓷 ${ }^{[21]}$ 及高熵陶瓷基复合材 料 ${ }^{[22-25]}$ 等相关研究陆续被报道。过渡金属碳化物和 喼化物具有高熔点、高硬度、优异的高温力学性能 和化学稳定性, 被广泛应用于航空航天领域 ${ }^{[26-28]}$, 并在核工业领域具有良好的应用前景 ${ }^{[29-30]}$ 。面对高 温、高应力、强辐照和强腐蚀等极端工作环境对性 能的要求, 通过高熵设计来提高陶瓷材料的性能是 目前研究的热点。

有研究报导, 高熵碳化物 (TaHfZrNb)C 陶瓷在 高温 $\left(1400\right.$ 和 $\left.1600{ }^{\circ} \mathrm{C}\right)$ 下的稳态蠕变速率约为其 中任意组成元素的二元碳化物陶瓷 $90 \%$ ，具有更高 的抗高温蠕变特性 ${ }^{[31]}$ 。高熵碳化物 $(\mathrm{HfTaZrNb}) \mathrm{C}$ 陶 瓷 ${ }^{[32]}$ 以及高熵二嗍化物 $(\mathrm{HfZrTaCrTi}) \mathrm{B}_{2}$ 、 $(H f M o Z r N b T i) B_{2}$ 、(HfMoTaNbTi)B ${ }_{2}$ 陶瓷都表现出比 其组成元素的二元化合物更高的硬度 ${ }^{[33]}$ 。高熵碳化 物(ZrTaNbTi)C 陶瓷用 $20 \mathrm{dpa}$ 剂量的 $3 \mathrm{MeV} \mathrm{Zr}$ 离子 辐照后，仍能保持晶格完整，晶胞膨胀仅 $0.2 \%$, 具 有良好的抗辐照损伤特性 ${ }^{[34]}$ 。然而高摘碳化物 (HfZrTaNbTi)C 由于阳离子亚晶格扭曲, 增强了声 子散射，导致其热导率低于其组元的二元碳化物 ${ }^{[5]}$ 。

高熵带来的性能提升或下降与其引起的微纳结 构变化有着十分密切的关系。透射电子显微镜(TEM) 能够在纳米及原子尺度给出材料的结构、元素分布 和价态信息，这有助于深刻理解高熵固溶结构的形 成机制以及结构与性能之间的内在关系。Zhang 等 ${ }^{[36]}$
通过 TEM 高分辨图像在三元合金 $\mathrm{CrCoNi}$ 中发现了 短程有序结构, 短程有序结构可以增大层错能并提 高硬度。Lei 等 ${ }^{[37]}$ 利用球差校正透射电子显微镜 (ACTEM)的环形明场像(ABF) 在高熵合金 TiZrHfNb 中发现了含氧化合物构成的纳米短程序结构, 改变 了位错剪切模式, 丰富了位错滑移体系, 增强了强 度和延展性。Ding 等 ${ }^{[38]}$ 利用 ACTEM 高角环形暗场 像 (HADDF) 结合 $\mathrm{X}$ 射线能谱发现在高熵合金 $\mathrm{CrMnFeCoNi}$ 和 $\mathrm{CrFeCoNiPd}$ 中存在浓度波/浓度振 荡，丰富了位错滑移体系，增大了层错能，在保持 硬度和延展性的情况下提高了强度。高熵合金的研 究展现出丰富的结构和性能特性, 但是, 从微结构 角度去探索高熵陶瓷中结构与性能相关性的研究 还很贵乏。高熵效应带来的优势本质上是基于微纳 结构的变化, 因此从高熵陶瓷微纳结构中揭示其性 能变化的内在原因和规律, 对获得有针对性的性能 改进方案以及探索更优性能的高熵陶瓷体系显得十 分重要。

本工作利用 TEM 和 ACTEM 对高熵嗍化物 (TiZrHfNbTa)B $B_{2}$ 和碳化物(TiZrHfNbTa)C 两种高熵 陶瓷进行纳米到原子尺度的结构表征和研究，探讨 高熵陶瓷细致微结构的信息特征, 揭示高熵组分设 计带来的独特的结构变化, 研究工作将为理解以性 能为导向的高熵陶瓷结构设计和构效关系提供实验 依据。

\section{1 实验方法}

\section{1 材料制备}

以自制高熵粉体为原料，利用放电等离子体 (SPS)烧结制备高熵嗍化物(TiZrHfNbTa) $\mathrm{B}_{2}$ 和碳化 物(TiZrHfNbTa)C 陶瓷。首先根据最终高熵陶瓷中 元素的等摩尔比计算金属氧化物的用量, 称取相应 的氧化物粉体 $\mathrm{TiO}_{2}(\geqslant 99.99 \%, \sim 0.1 \mu \mathrm{m}), \mathrm{ZrO}_{2}$ 
$(\geqslant 99.95 \%, \sim 0.2 \mu \mathrm{m}), \mathrm{HfO}_{2}(\geqslant 99.95 \%, \sim 0.2 \mu \mathrm{m})$, $\mathrm{Nb}_{2} \mathrm{O}_{5}(\geqslant 99.8 \%, \sim 2 \mu \mathrm{m}), \mathrm{Ta}_{2} \mathrm{O}_{5}(\geqslant 99.5 \%, \sim 1.5 \mu \mathrm{m})$ 与石墨 $(\geqslant 99.9 \%, \sim 40 \mathrm{~nm})$ 或 $\mathrm{B}_{4} \mathrm{C}(\sim 0.3 \mu \mathrm{m})$, 然后以 乙醇为溶剂, $\mathrm{WC}$ 球为研磨介质, 球与粉体的质量比 为 $6: 1$, 以 $560 \mathrm{r} / \mathrm{min}$ 的转速球磨 $10 \mathrm{~h}$ 。对球磨获得 的混合物浆料干燥并过篎, 再将混合物粉末压制成 圆片, 放入石墨坩埚中进行硼热或者碳热还原合 成。高熵化合物粉末的合成条件为真空气氛, 升温 速率为 $10{ }^{\circ} \mathrm{C} / \mathrm{min}$, 在 $1600{ }^{\circ} \mathrm{C}$ 保温 $0.5 \mathrm{~h}$ (ZT-15-20 石墨加热炉, 上海辰华科技有限公司), 然后将合成 的粉末过篮 $(200$ 目, $74 \mu \mathrm{m})$ 。过篮后的高熵粉末放入 石墨模具中, 采用 SPS(HP D25, FCT, 德国)烧结, 烧结条件为真空气氛, 压力 $50 \mathrm{MPa}$, 以 $100{ }^{\circ} \mathrm{C} / \mathrm{min}$ 的升温速率升至 $1700{ }^{\circ} \mathrm{C}$ 保温 $10 \mathrm{~min}$ 。材料的详细制 备工艺可以参考文献[9,22-23]。

\section{2 微观结构表征}

对制备好的块体进行切片、研磨、抛光，制成 厚度约 $40 \mu \mathrm{m}$ 的薄片。再利用 $5 \mathrm{kV} \mathrm{Ar}$ 离子进行离 子减薄(PIPS II 695, Gatan, USA), 获得可用于 TEM 分析的样品。利用透射电子显微镜(TEM, FEI Tecnai G2 F20, Japan)对样品纳米尺度的结构进行表征, 然 后用配备有双能谱(EDS, Oxford X-Max, UK)的球差 校正电镜(ACTEM, Hitachi HF5000, Japan)对样品原 子尺度的结构进行表征。

\section{2 结果与讨论}

\section{1 纳米尺度的结构信息}

对于高熵多元化合物陶瓷，不同过渡金属原子 的扩散速率不尽相同，在烧结过程中很可能由于扩 散程度不同而导致成分偏析，局部成分偏析又会由 于热膨胀系数不同而导致热残余应力。若成分偏析 存在规律性则会形成长周期结构, 热残余应力则会 促使位错等缺陷的生长。对于六方和面心立方结构, 其密排面分别是 $(0001)$ 和 $\{111\}$, 是最容易形成位错 的晶面。如果出现长周期结构, 也很可能存在习惯 生长面。通过不同晶带轴的 TEM 表征, 可以获得相 关信息。针对密排六方结构的高熵硼化物 (TiZrHfNbTa)B B $_{2}$ 和面心立方结构的高摘碳化物 (TiZrHfNbTa)C 陶瓷可能存在的上述结构和微结构, 本课题组开展了细致的 TEM 表征和分析。

通过 TEM 明场像可以看到高熵(TiZrHfNbTa)B $B_{2}$ 和(TiZrHfNbTa)C 陶瓷由微米级的晶粒构成, 分别 如图 1(a, d, g) 和图 2(a,d, g) 所示, 单颗晶粒的祄度 均匀, 没有位错和孪晶等结构缺陷。对高熵

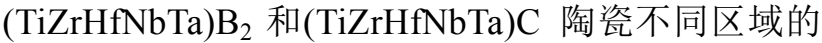
晶粒做了 $[1 \overline{2} 1 \overline{3}] 、[\overline{2} 4 \overline{2} 3] 、[01 \overline{1} 1]$ 和 [011]、[001]、[112] 带轴的选区电子衍射(SAD)测试，倒易点阵呈现完 整的单晶衍射花样(如图 1(b,e, h) 和图 2(b,e, h) 所 示), 可以判断(TiZrHfNbTa)B B $_{2}$ 和(TiZrHfNbTa)C 分 别具有六方和面心立方结构。其中衍射斑点没有拉 长，显示出良好的结晶性且没有结构缺陷; 没有发 现卫星斑点或超点阵斑点, 说明不同过渡金属元素 随机地固溶在晶格当中, 并没有形成某种元素的长 程有序分布。相应的高分辨图像呈现较完整的晶格 条纹(如图 1(c, f, i) 和图 2(c, f, i) 所示), 同样说明高 熵多元固溶化合物的晶格完整性。

通过几何相位分析法(GPA)可以获得结构应变 场大小等信息, 准确度可达 $0.003 \mathrm{~nm}^{[39]}$ 。通过对 $[1 \overline{2} 1 \overline{3}]$ 晶带轴获得的(TiZrHfNbTa) $B_{2}$ 高分辨 TEM 图 像(图 3(a))和[011]晶带轴获得的(TiZrHfNbTa)C 高 分辨 TEM 图像(图 3(e))进行 GPA 分析分别得到结构 应变场图像，如图 3(b d) 和图 3(f h) 所示，从中可以 看到整个区域被红色(正应变)和绿色(负应变)覆盖, 对比应变大小的标尺, 说明 (TiZrHfNbTa) $\mathrm{B}_{2}$ 和 (TiZrHfNbTa)C 都存在纳米尺度的结构畸变。图 $3(b \sim d)$ 中的正应变和负应变交叉点部分集中在右侧 区域，而图 3(f h h)中的应变交叉点分布相对均匀一 些。由此可见，多元过渡金属固溶形成的高熵陶瓷 存在一定的结构畸变, 且会存在应变不均的情况。 内应力的大小是否会因为固溶元素的不同而有所区 别，是否会改变位错的滑移机制，从而影响材料的 力学性能, 都是亟待解决的科学问题。

\section{2 原子尺度的结构信息}

由于高熵陶瓷中固溶的各种金属原子的半径存 在差异，在形成高熵相时，原子离散度对于不同固 溶元素存在差别, 因此金属元素原子离散度可以通 过 ACTEM 中的 HAADF 原子像进行观察, 然后通 过 StatSTEM 软件获得原子坐标进行量化处理 ${ }^{[40]}$ 。 高熵嗍化物(TiZrHfNbTa)B ${ }_{2}$ 和碳化物(TiZrHfNbTa)C 陶瓷的 HADDF 原子相如图 4(a,d)所示。通过 StatSTEM 中的高斯拟合获得相应原子坐标, 由此 计算得出 $(\mathrm{TiZrHfNbTa}) \mathrm{B}_{2}$ 和 $(\mathrm{TiZrHfNbTa}) \mathrm{C}$ 的原子 离散度分别是 $d 1=0.00101 \mathrm{~nm}, d 2=0.00056 \mathrm{~nm}$ 和 $d 1=0.00109 \mathrm{~nm}, d 2=0.00204 \mathrm{~nm}$ 。如图 4(b,c,e,f)所示, 其中, $d 1$ 和 $d 2$ 分别为 $x$ 方向和 $y$ 方向的原子间距，将 晶格间距由小到大用绿色到橘色标注，颜色变化差 异直观地表现原子离散度的分布情况。由此可见, 虽有不同原子半径的过渡金属元素固溶，但离散度 较小, 说明这几种元素很好地固溶在晶格当中。 

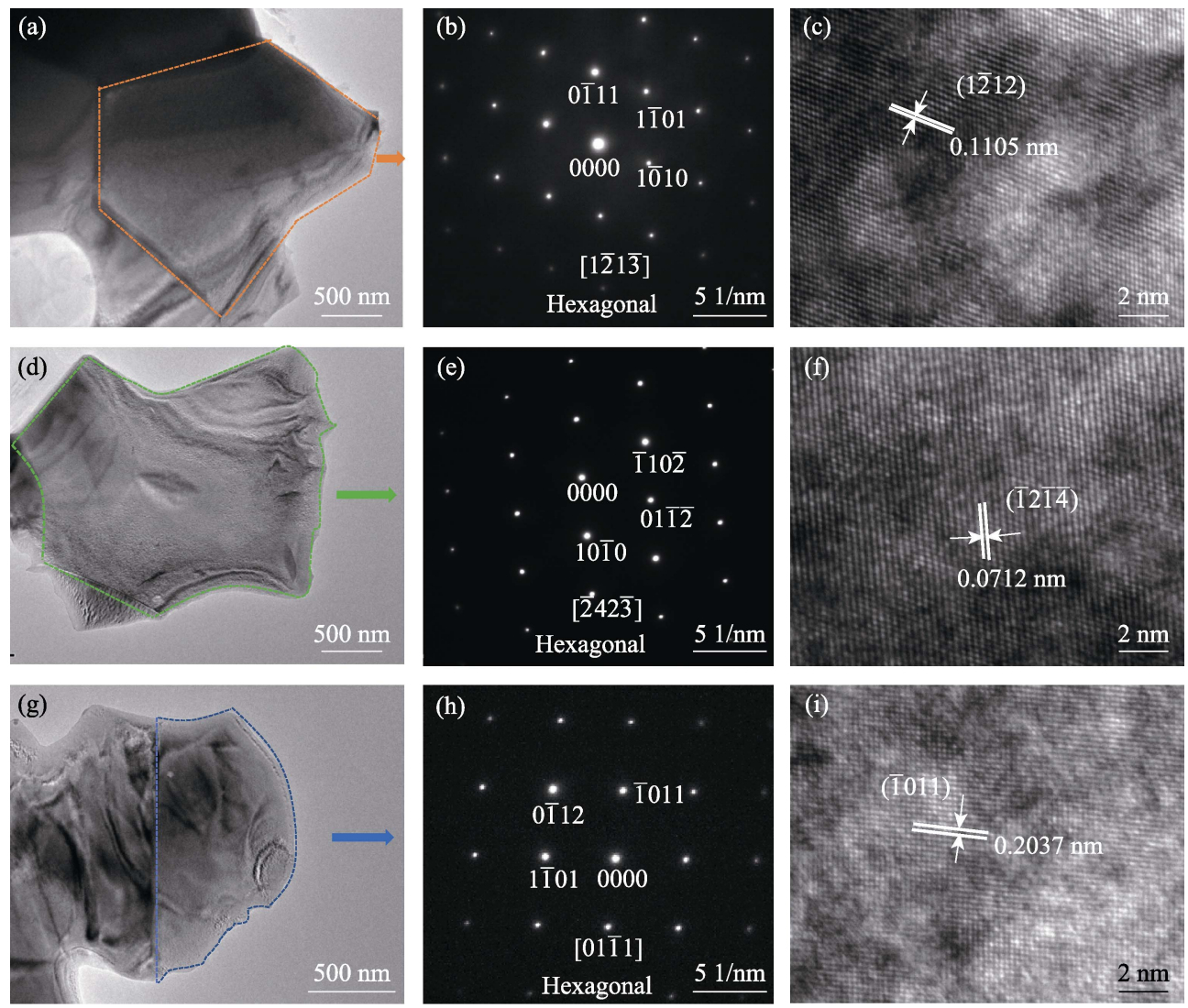

图 1 (TiZrHfNbTa) $B_{2}$ 陶瓷的 TEM 明场像 $(\mathrm{a}, \mathrm{d}, \mathrm{g})$, 相应晶粒的 SAD 花样 $(\mathrm{b}, \mathrm{e}, \mathrm{h})$ 和高分辨图像 $(\mathrm{c}, \mathrm{f}, \mathrm{i})$

Fig. 1 Bright field TEM images (a, d, g), SAD patterns (b, e, h) and high-resolution images (c, f, i) of the (TiZrHfNbTa) $\mathrm{B}_{2}$ ceramic
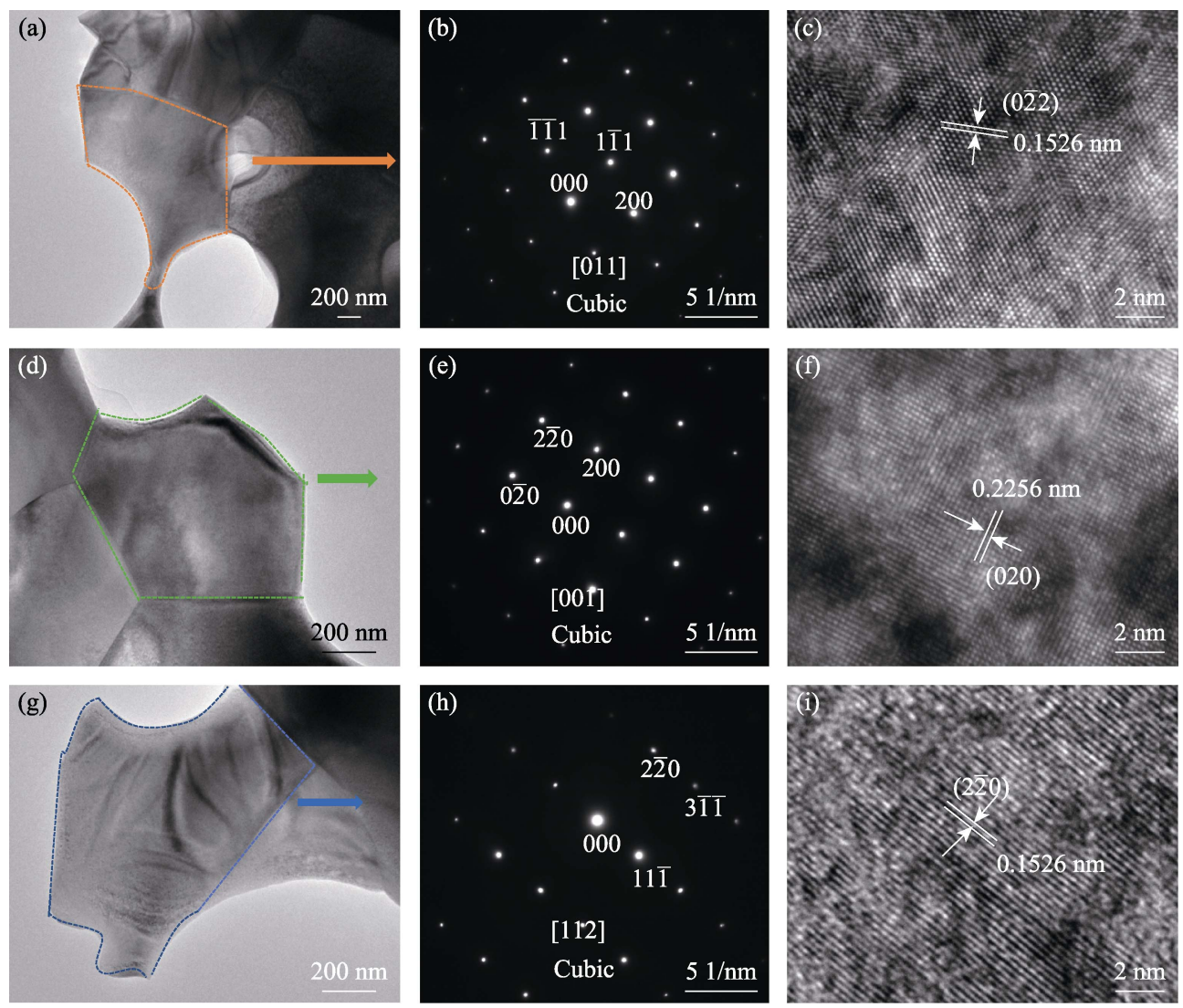

图 2 (TiZrHfNbTa)C 陶瓷的 TEM 明场像 $(\mathrm{a}, \mathrm{d}, \mathrm{g})$, 相应晶粒的 SAD 花样 $(\mathrm{b}, \mathrm{e}, \mathrm{h})$ 和高分辨图像 $(\mathrm{c}, \mathrm{f}, \mathrm{i})$

Fig. 2 Bright field TEM images (a, d, g), SAD patterns $(b, e, h)$ and high-resolution images (c, $f, i)$ of the (TiZrHfNbTa)C ceramic 

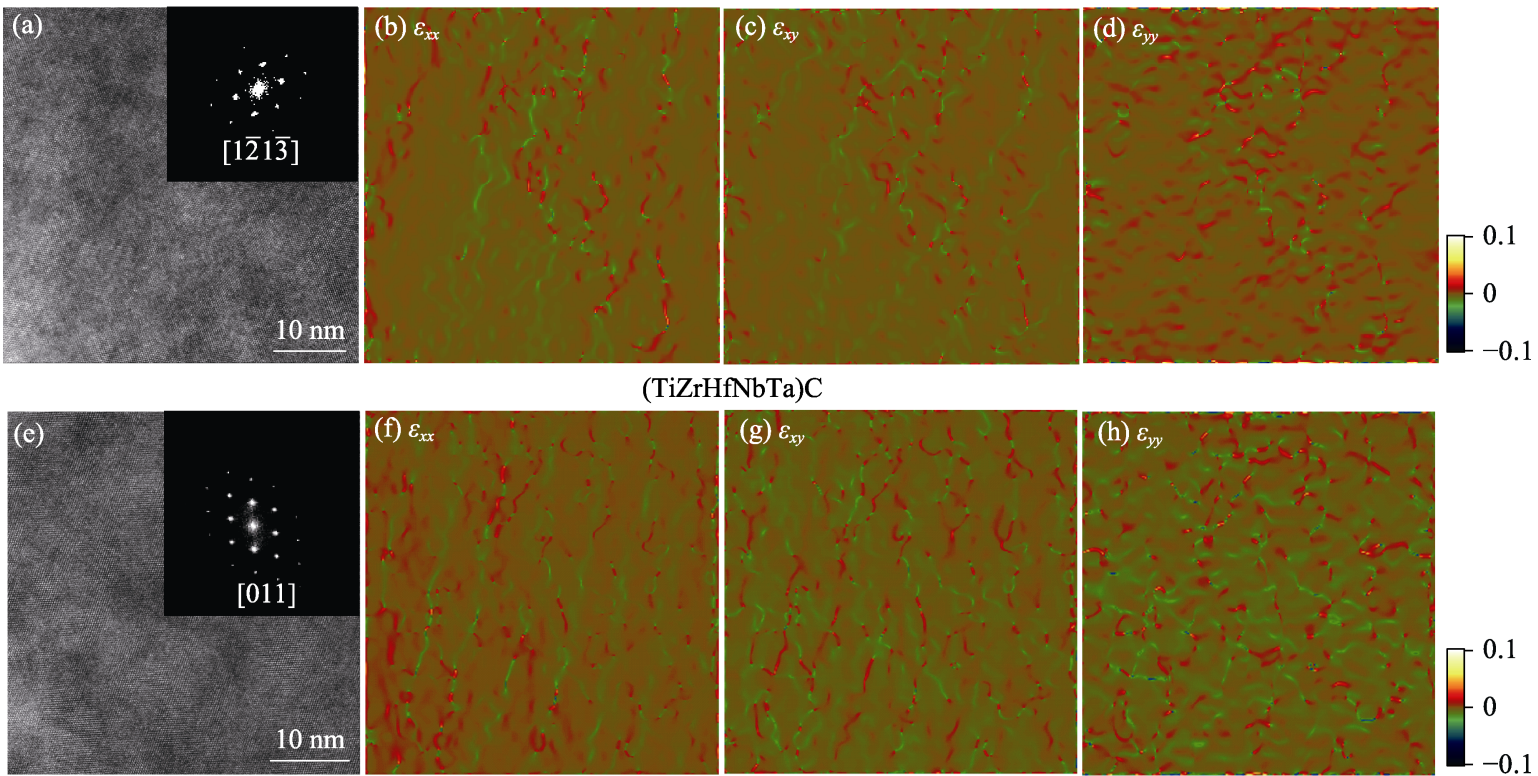

图 3 (TiZrHfNbTa)B $\mathrm{B}_{2}(\mathrm{a})$ 和(TiZrHfNbTa)C(e)的高分辨 TEM 图像，右上角插图是相应的傅里叶变换的衍射花样; $(\mathrm{TiZrHfNbTa}) \mathrm{B}_{2}(\mathrm{~b} \sim \mathrm{d})$ 和(TiZrHfNbTa)C(f $\left.\sim \mathrm{h}\right)$ 样品 GPA 处理的不同方向上的应力分布图

Fig. 3 High-resolution TEM images of (TiZrHfNbTa)B $\mathrm{B}_{2}$ (a) and (TiZrHfNbTa)C (e) with SAD patterns inserted in the upper right corner; and corresponding phase images of (TiZrHfNbTa) $\mathrm{B}_{2}(\mathrm{~b}-\mathrm{d})$ and $(\mathrm{TiZrHfNbTa}) \mathrm{C}(\mathrm{f}-\mathrm{h})$ in different directions processed by GPA
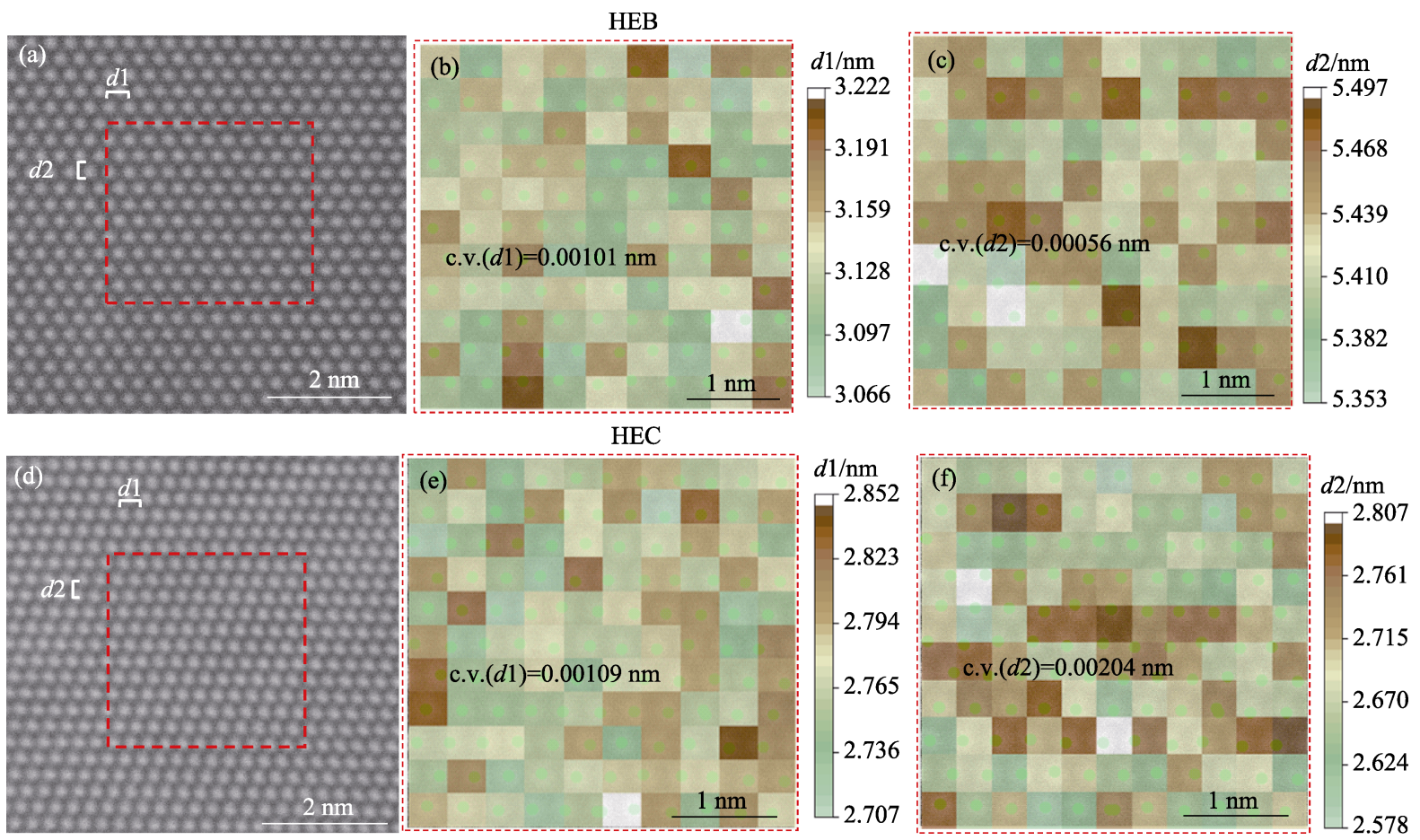

图 4 ( TiZrHfNbTa)B ${ }_{2}(\mathrm{a})$ 和(TiZrHfNbTa)C(d)的 HAADF 图像及其相应的原子离散分布图 $((\mathrm{b}, \mathrm{c})$ 和 $(\mathrm{e}, \mathrm{f}))$

Fig. 4 HAADF images of (TiZrHfNbTa)B $\mathrm{B}_{2}$ (a) and (TiZrHfNbTa)C (d); Corresponding atomic discrete distribution maps $((\mathrm{b}, \mathrm{c})$ and $(\mathrm{e}, \mathrm{f}))$

原子离散度反映的是金属阳离子的原子结构信息， 在一定程度上反映了晶格应力的大小; 而高分辨成 像包含了阴阳离子整体效应的结构信息，结合原子 离散度和 GPA 分析可以很清晰地得到局部区域的 原子分布及其结构应变信息。

通过 HAADF 原子像及其 EDS 面分布可以清晰
地看到各个过渡元素在局部区域的分布，然而 B 和 $\mathrm{C}$ 的元素信号容易被样品吸收, 很难通过 EDS 获得 确切的分布信息, 如图 5 所示。通过过渡元素的 EDS 面分布表征, 可以发现在高熵(TiZrHfNbTa)B $\mathrm{B}_{2}$ 和 (TiZrHfNbTa)C 陶瓷中存在纳米尺度浓度分布不均 匀的现象, 而且这种浓度波动没有规律性。类似现 

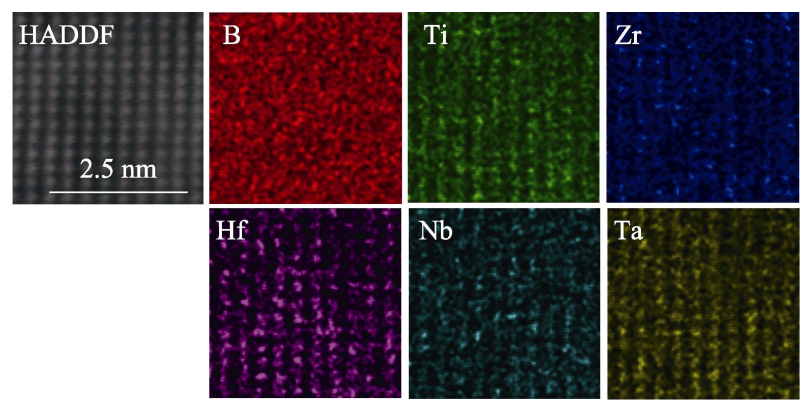

(a) (TiZrHfNbTa) $B_{2}$
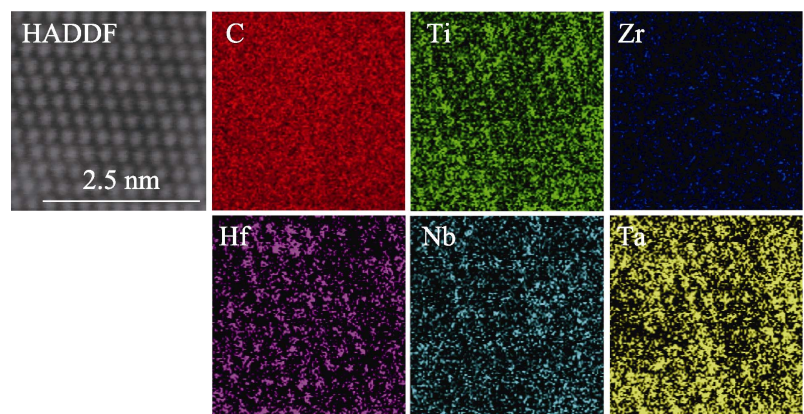

(b) $(\mathrm{TiZrHfNbTa}) \mathrm{C}$

图 5 (TiZrHfNbTa)B $\mathrm{B}_{2}(\mathrm{a})$ 和 (TiZrHfNbTa)C(b) 的原子级 HADDF 图像及其 EDS 面分布

Fig. 5 Atomic HADDF images and corresponding EDS mappings of (TiZrHfNbTa)B $\mathrm{B}_{2}$ (a) and (TiZrHfNbTa)C (b)

象在其它高熵陶瓷的研究中也有所发现 ${ }^{[41-42]}$ 。这种 浓度波动或者类似的原子分布不均匀的现象, 将减 小按照均匀分布计算的混合熵数值。如果在高摘陶 瓷中普遍存在这种现象, 从热力学角度来看, 则会 减弱熵稳定的陶瓷体系的“熵稳定”效果。

\section{3 结论}

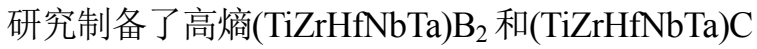
陶瓷，并利用 TEM 和 ACTEM 对其进行了纳米和原 子尺度的结构表征。研究结果发现, 高熵陶瓷保持 了单一晶体结构的完整性和微观尺度的固溶元素分 布均匀性, 没有结构缺陷或固溶元素的长程有序分 布。但多元过渡元素的固溶会造成一定大小的晶格 应力和原子离散, 并在晶格中的分布存在纳米尺度 的浓度不均匀。本工作获得了高熵硼化物和高熵碳 化物的固溶结构和微结构特征, 据此可以开展相关 理论计算以明确固溶结构稳定性的热力学机制, 并 通过相关物理性能的表征(包括硬度、强度、韧性和 抗辐照损伤行为), 探讨高熵结构和性能之间的内 在关系。

\section{参考文献:}

[1] GEORGE E P, RAABE D, RITCHIE R O. High-entropy alloys.
Nature Reviews Materials, 2019, 4(8): 515-534.

[2] ROST C M, SACHET E, BORMAN T, et al. Entropy-stabilized oxides. Nature Communications, 2015, 6(8): 8485.

[3] ZHOU L, LI F, LIU J X, et al. High-entropy thermal barrier coating of rare-earth zirconate: a case study on $\left(\mathrm{La}_{0.2} \mathrm{Nd}_{0.2} \mathrm{Sm}_{0.2} \mathrm{Eu}_{0.2} \mathrm{Gd}_{0.2}\right)_{2} \mathrm{Zr}_{2} \mathrm{O}_{7}$ prepared by atmospheric plasma spraying. Journal of the European Ceramic Society, 2020, 40(15): 5731-5739.

[4] LI F, ZHOU L, LIU J X, et al. High-entropy pyrochlores with low thermal conductivity for thermal barrier coating materials. Journal of Advanced Ceramics, 2019, 8(4): 576-582.

[5] WRIGHT A J, WANG Q, KO S T, et al. Size disorder as a descriptor for predicting reduced thermal conductivity in mediumand high-entropy pyrochlore oxides. Scripta Materialia, 2020, 181: $76-81$.

[6] REN K, WANG Q, SHAO G, et al. Multicomponent high-entropy zirconates with comprehensive properties for advanced thermal barrier coating. Scripta Materialia, 2020, 178: 382-386.

[7] ZHAO Z, XIANG H, DAI F Z, et al. $\left(\mathrm{La}_{0.2} \mathrm{Ce}_{0.2} \mathrm{Nd}_{0.2} \mathrm{Sm}_{0.2} \mathrm{Eu}_{0.2}\right)_{2} \mathrm{Zr}_{2} \mathrm{O}_{7}$ : a novel high-entropy ceramic with low thermal conductivity and sluggish grain growth rate. Journal of Materials Science \& Technology, 2019, 35(11): 2647-2651.

[8] GILD J, ZHANG Y, HARRINGTON T, et al. High-entropy metal diborides: a new class of high-entropy materials and a new type of ultrahigh temperature ceramics. Scientific Reports, 2016, 6: 37946.

[9] LIU J X, SHEN X Q, WU Y, et al. Mechanical properties of hot-pressed high-entropy diboride-based ceramics. Journal of Advanced Ceramics, 2020, 9(4): 503-510.

[10] ZHAO P B, ZHU J P, ZHANG Y L, et al. A novel high-entropy monoboride $\left(\mathrm{Mo}_{0.2} \mathrm{Ta}_{0.2} \mathrm{Ni}_{0.2} \mathrm{Cr}_{0.2} \mathrm{~W}_{0.2}\right) \mathrm{B}$ with superhardness and low thermal conductivity. Ceramics International, 2020, 46(17): 26626-26631.

[11] CHEN H, ZHAO Z, XIANG H, et al. Effect of reaction routes on the porosity and permeability of porous high entropy $\left(\mathrm{Y}_{0.2} \mathrm{Yb}_{0.2} \mathrm{Sm}_{0.2} \mathrm{Nd}_{0.2} \mathrm{Eu}_{0.2}\right) \mathrm{B}_{6}$ for transpiration cooling. Journal of Materials Science \& Technology, 2020, 38: 80-85.

[12] WEI X F, LIU J X, LI F, et al. High entropy carbide ceramics from different starting materials. Journal of the European Ceramic Society, 2019, 39(10): 2989-2994.

[13] WEI X F, QIN Y, LIU J X, et al. Gradient microstructure development and grain growth inhibition in high-entropy carbide ceramics prepared by reactive spark plasma sintering. Journal of the European Ceramic Society, 2020, 40(4): 935-941.

[14] DAI F Z, WEN B, SUN Y, et al. Theoretical prediction on thermal and mechanical properties of high entropy $\left(\mathrm{Zr}_{0.2} \mathrm{Hf}_{0.2} \mathrm{Ti}_{0.2} \mathrm{Nb}_{0.2} \mathrm{Ta}_{0.2}\right) \mathrm{C}$ by deep learning potential. Journal of Materials Science \& Technology, 2020, 43: 168-174.

[15] WANG K, CHEN L, XU C, et al. Microstructure and mechanical properties of (TiZrNbTaMo)C high-entropy ceramic. Journal of Materials Science \& Technology, 2020, 39: 99-105.

[16] QIN Y, LIU J X, LI F, et al. A high entropy silicide by reactive spark plasma sintering. Journal of Advanced Ceramics, 2019, 8(1): $148-152$.

[17] QIN Y, WANG J C, LIU J X, et al. High-entropy silicide ceramics developed from (TiZrNbMoW)Si $i_{2}$ formulation doped with aluminum. Journal of the European Ceramic Society, 2020, 40(8): 2752-2759.

[18] GILD J, BRAUN, J KAUFMANN K, et al. A high-entropy silicide: $\left(\mathrm{Mo}_{0.2} \mathrm{Nb}_{0.2} \mathrm{Ta}_{0.2} \mathrm{Ti}_{0.2} \mathrm{~W}_{0.2}\right) \mathrm{Si}_{2}$. J. Materiomics, 2019, 5(3): 337-343.

[19] BAO W C, WANG X G, DING H J, et al. High-entropy $\mathrm{M}_{2}$ AlC-MC 
$(\mathrm{M}=\mathrm{Ti}, \mathrm{Zr}, \mathrm{Hf}, \mathrm{Nb}, \mathrm{Ta})$ composite: synthesis and microstructures. Scripta Materialia, 2020, 183: 33-38.

[20] LI Y B, LU J, LI M, et al. Multielemental single atom-thick A layers in nanolaminated $\mathrm{V}_{2}(\mathrm{Sn}, \mathrm{A}) \mathrm{C}(\mathrm{A}=\mathrm{Fe}, \mathrm{Co}, \mathrm{Ni}, \mathrm{Mn})$ for tailoring magnetic properties. Proceedings of the National Academy of Sciences of the United States of America, 2020, 117(2): 820-825.

[21] LI F, BAO W C, SUN S K, et al. Synthesis of single-phase metal oxycarbonitride ceramics. Scripta Materialia, 2020, 176: 17-22.

[22] LU K, LIU J X, WEI X F, et al. Microstructures and mechanical properties of high-entropy $\left(\mathrm{Ti}_{0.2} \mathrm{Zr}_{0.2} \mathrm{Hf}_{0.2} \mathrm{Nb}_{0.2} \mathrm{Ta}_{0.2}\right) \mathrm{C}$ ceramics with the addition of $\mathrm{SiC}$ secondary phase. Journal of the European Ceramic Society, 2020, 40(54): 1839-1847.

[23] SHEN X Q, LIU J X, LI F, et al. Preparation and characterization of diboride-based high entropy $\left(\mathrm{Ti}_{0.2} \mathrm{Zr}_{0.2} \mathrm{Hf}_{0.2} \mathrm{Nb}_{0.2} \mathrm{Ta}_{0.2}\right) \mathrm{B}_{2}$-SiC particulate composites. Ceramics International, 2019, 45(18): 24508-24514.

[24] QIN M, GILD J, HU C, et al. Dual-phase high-entropy ultra-high temperature ceramics. Journal of the European Ceramic Society, 2020, 40(15): 5037-5050.

[25] ZHANG Y, SUN S K, GUO W M, et al. Optimal preparation of high-entropy boride-silicon carbide ceramics. Journal of Adcanced Ceramics, 2020, 10: 173-180.

[26] CHEN L, WANG K, SU W T, et al. Research progress of transition metal non-oxide high-entropy ceramics. Journal of Inorganic Materials, 2020, 35(7): 748-758.

[27] WANG X G, ZHANG G J, ZHAO J, et al. High-strength ZrC ceramics doped with aluminum. Journal of the American Ceramic Society, 2014, 97(11): 3367-3370.

[28] YE B, WEN T, CHU Y, et al. High-temperature oxidation behavior of $\left(\mathrm{Hf}_{0.2} \mathrm{Zr}_{0.2} \mathrm{Ta}_{0.2} \mathrm{Nb}_{0.2} \mathrm{Ti}_{0.2}\right) \mathrm{C}$ high-entropy ceramics in air. Journal of the American Ceramic Society, 2020, 103(1): 500-507.

[29] BAO W C, LIU J X, WANG X G, et al. Structural evolution in $\mathrm{ZrC}-\mathrm{SiC}$ composite irradiated by $4 \mathrm{MeV}$ Au ions. Nuclear Instruments \& Methods in Physics Research Section B-Beam Interactions with Materials and Atoms, 2018, 434: 23-28.

[30] BAO W C, ROBERTSON S, LIU J X, et al. Structural integrity and characteristics at lattice and nanometre levels of $\mathrm{ZrN}$ polycrystalline irradiated by $4 \mathrm{MeV} \mathrm{Au}$ ions. Journal of the European
Ceramic Society, 2018, 38(13): 4373-4383.

[31] HAN X X, GIRMAN V, SEDLAK R, et al. Improved creep resistance of high entropy transition metal carbides. Journal of the European Ceramic Society, 2020, 40(7): 2709-2715.

[32] CASTLE E, CSANADI T, GRASSO S, et al. Processing and properties of high-entropy ultra-high temperature carbides. Scientific Reports, 2018, 8: 8609.

[33] ZHANG Y, GUO W M, JIANG Z B, et al. Dense high-entropy boride ceramics with ultra-high hardness. Scripta Materialia, 2019, 164: $135-139$.

[34] WANG F, YAN X L, WANG T Y, et al. Irradiation damage in $\left(\mathrm{Zr}_{0.25} \mathrm{Ta}_{0.25} \mathrm{Nb}_{0.25} \mathrm{Ti}_{0.25}\right) \mathrm{C}$ high-entropy carbide ceramics. Acta Materialia, 2020, 195: 739-749.

[35] YAN X L, CONSTANTIN L, LU Y F, et al. $\left(\mathrm{Hf}_{0.2} \mathrm{Zr}_{0.2} \mathrm{Ta}_{0.2} \mathrm{Nb}_{0.2} \mathrm{Ti}_{0.2}\right) \mathrm{C}$ high-entropy ceramics with low thermal conductivity. Journal of the American Ceramic Society, 2018, 101(10): 4486-4491.

[36] ZHANG R P, ZHAO S T, DING J, et al. Short-range order and its impact on the CrCoNi medium-entropy alloy. Nature, 2020, 581(7808): $283-287$.

[37] LEI Z F, LIU X J, WU Y, et al. Enhanced strength and ductility in a high-entropy alloy via ordered oxygen complexes. Nature, 2018, 563(7732): 546-550.

[38] DING Q Q, ZHANG Y, CHEN X, et al. Tuning element distribution, structure and properties by composition in high-entropy alloys. Nature, 2019, 574(7777): 223-227.

[39] HYTCH M J, PUTAUX J L, PENISSON J M. Measurement of the displacement field of dislocations to 0.03 angstrom by electron microscopy. Nature, 2003, 423(6937): 270-273.

[40] BACKER A D, BOS K H W V D, BROEK W V D, et al. StatSTEM: an efficient approach for accurate and precise modelbased quantification of atomic resolution electron microscopy images. Ultramicroscopy 2016, 171: 104-116.

[41] DUSZA J, SVEC P, GIRMAN V, et al. Microstructure of (Hf-Ta-Zr-Nb)C high-entropy carbide at micro and nano/atomic level. Journal of the European Ceramic Society, 2018, 38(12): 4303-4307.

[42] CSANADI T, CASTLE E, REECE M J, et al. Strength enhancement and slip behaviour of high-entropy carbide grains during microcompression. Scientific Reports, 2019, 9: 10200. 